\title{
Plasma radiation studies in Magnum-PSI using resistive bolometry
}

\author{
G.G. van Eden*1, M.L. Reinke ${ }^{2}$, S. Brons ${ }^{1}$, G. van der Bijl ${ }^{1}$, B. \\ Krijger $^{1}$, R. Lavrijsen ${ }^{3}$, S.P. Huber ${ }^{4}$, R. Perillo ${ }^{1}$, M.C.M. van \\ de Sanden $^{1}$, T.W. Morgan ${ }^{1}$ \\ ${ }^{1}$ DIFFER - Dutch Institute for Fundamental Energy Research, De Zaale 20, \\ 5612 AJ Eindhoven, the Netherlands \\ ${ }^{2}$ Oak Ridge National Laboratory, Oak Ridge, TN 37831, USA \\ ${ }^{3}$ Department of Applied Physics, Center for NanoMaterials and COBRA \\ Research Institute, Eindhoven University of Technology, PO Box 513, 5600 \\ MB Eindhoven, The Netherlands \\ ${ }^{4}$ École Polytechnique Fédérale de Lausanne, Station 9, 1015 Lausanne, \\ Switzerland \\ E-mail: $g \cdot g \cdot v a n e d e n @ d i f f e r . n l$
}

\begin{abstract}
.
Both the physics of divertor detachment and vapour shielding are characterized by a relatively large amount of radiation produced in the divertor. The linear plasma generator Magnum-PSI is well-suited to study such processes due to its ITER-divertor relevant plasma conditions, simplified geometry and diagnostic accessibility. The need the quantify the plasma radiated power close to the target surface motivated the development of a 4-channel resistive bolometer for Magnum-PSI, and marks the first deployment of such a diagnostic on a linear device. An axially resolved measurement of plasma emission at arbitrary distances from the target surface is now possible. The radial position of the detector can be varied, hereby viewing the full diameter of the plasma column or down to a central region. The overall system design is discussed alongside a comparison of the spectral absorbance of carbon-coated versus non-coated $\mathrm{Au} / \mathrm{Al}$ bolometer sensors. Despite low electron temperatures of the plasma (1-5 eV), the observed power densities were found to be 10-37 times the sensor noise floor of $\sim 0.1 \mathrm{~W} \mathrm{~m}^{-2}$. A synthetic diagnostic based on collisional radiative model calculations from ADAS could well match observed values from $\mathrm{H}$ and Ne plasmas while the measured values for $\mathrm{Ar}$ and He were more difficult to reproduce. The obtained findings allow for approximate power balance calculations in Magnum-PSI indicating that maximally $\sim 47 \%$ and $\sim 14 \%$ of the total power is lost by radiation in the cases of $\mathrm{Ar}$ and $\mathrm{Ne} / \mathrm{He}$ respectively. The results demonstrate the feasibility of resistive bolometry in low temperature high density plasma regions and on long timescales $(>450 \mathrm{~s})$ which is of relevance to ITER. Due to long-term temperature drifts which were observed, a planned upgrade involves the installation of a shutter and FPGAbased electronics for increased accuracy.
\end{abstract}




\section{Introduction}

The power entering the scrape-off-layer (SOL) in future fusion reactors such as DEMO is expected to be several times larger than the predicted levels for ITER [1]. Ensuring the integrity and successful long-term heat handling performance of the tungsten (W) ITER divertor over time is challenging, making a solution for DEMO even more difficult to attain. In DEMO, reducing the exhaust power to acceptable divertor heat loads is foreseen through strong radiative dissipation in SOL and divertor [2]. Any potential loss of control over this scheme is however still likely to result in divertor heat fluxes exceeding limits which can be safely tolerated, hereby potentially damaging components $[3]$.

Operation in semi- or full-detached divertor plasma scenarios are envisioned to mitigate this issue [4]. Here seeding impurities will be introduced which will radiate predominantly in the plasma edge and SOL to reduce the parallel heat flux prior to arrival at the divertor surface [5]. From the material side, liquid metals are considered for DEMO as a plasma facing material in the divertor [6].

Such materials may further aid in increasing the damage tolerance in response to high heat loads since the material can be self-replenishing [7] and self-protecting once the vapour shielding regime is entered where evaporated impurities act as a partial barrier between the plasma and the liquid surface $[8,9]$. In both plasma detachment and vapour shielding, the incoming plasma energy is partially converted into radiation and neutral particles which are spread over a wider area, hence reducing the local target heat flux.

Quantifying the amount of power dissipated via radiation in the divertor SOL is identified as a key parameter in both detachment and vapour shielding studies but it is unclear how this extrapolates to reactor designs. Particularly steady-state radiated power loss produced close to the target surface, as is the case during vapour shielding, has not been studied in detail up to now.

The Magnum-PSI linear plasma generator $[10,11]$ offers the capability of replicating ITER divertor target conditions and a parallel heat flux $\left(\mathrm{q}_{\|}\right)$up to $\sim 20 \mathrm{MW} \mathrm{m}^{-2}$ while allowing for ease of diagnostic access, controllable conditions and simplicity of the plasma beam geometry compared to full-scale tokamak tests. While also the neutral pressure during operation can be varied in the expected ITER range (1-10 Pa) [12], the parallel heat transport of a few hundred $\mathrm{MW} \mathrm{m}^{-2}$ in ITER [3] cannot be fully replicated which impacts the feasibility of investigating the complete physics of detachment. Despite this, linear plasma generators offer a useful platform in validating radiation modelling for low-temperature, high-density plasmas with a variable background pressure, to address important questions related to detachment physics [13].

Current experimental campaigns in Magnum-PSI are aimed at understanding the physics occurring during the change from sheath-limited to detached plasma regimes, similar to previous studies in Pilot-PSI [14]. Also, extensive campaigns investigating the power handling capabilities of liquid $\mathrm{Sn}$ and Li plasma facing components (PFCs) 
and the physics of liquid metal vapour shielding have been performed in both machines $[8,9]$. However, no means of a direct measurement of the radiated power loss from the plasma had been available up to now.

The need to quantify radiated power loss at arbitrary distances from the target surface motivated the development of a bolometry system [15] for Magnum-PSI. Despite a lower time resolution and overall sensitivity, a resistive bolometer system was chosen due to its approximately flat response over the plasma photon energy range and superior long-term sensitivity stability as compared to e.g. AXUV diodes [16]. Since the Magnum-PSI plasma volume is much smaller $(\mathrm{FWHM} \approx 20 \mathrm{~mm}$ ) than e.g. (divertor) tokamak plasmas and the electron temperature $\left(T_{\mathrm{e}}\right)$ is typically only a few $\mathrm{eV}$, the line-integrated emissivity was expected to be low, potentially even below the typical resistive bolometer noise-equivalent-power density of $\sim 0.1 \mathrm{~W} \mathrm{~m}^{-2}$ [17]. However, initial modelling of a synthetic diagnostic and a collisional radiative model using the ADAS package [18] simulating the Magnum-PSI plasma yielded a signal-to-noise ratio (SNR) $>5$, providing confidence in the feasibility of resolving the radiated power from the plasma above the instrument noise.

A 4-channel resistive metal foil bolometer system viewing the plasma beam perpendicularly, close to the target region, has hence been designed and installed. Two channels have been provided with a thin layer of deposited carbon to increase absorption in the visual wavelength range [19], with two left uncoated for comparison purposes. The hardware design and spectral response of the Magnum-PSI bolometry system are described. Measurements of steady-state radiated power loss close to the target surface in Magnum-PSI are reported for the first time, showing challenges associated with longpulse measurements. Successful bolometry operation is further demonstrated during divertor-like detachment studies, impurity seeding experiments and volumetric plasma emission tests. The SNR during operation in $\mathrm{H}, \mathrm{He}, \mathrm{Ne}$ and $\mathrm{Ar}$ at a range of plasma conditions and the fraction of radiation in the overall power balance are presented.

\section{Methods}

\subsection{Magnum-PSI}

The Magnum-PSI linear plasma device utilizes a wall-stabilized cascaded arc source [20] to produce a low temperature $(1-5 \mathrm{eV})$, high-density $\left(\sim 10^{20} \mathrm{~m}^{-3}\right)$ plasma. A superconducting magnet produces a variable magnetic field strength up to $2.5 \mathrm{~T}$, to confine the plasma into a beam (FWHM $\approx 20 \mathrm{~mm}$ ) allowing for steady-state exposures of target materials in ITER-relevant conditions [21]. The background pressure near the target can be reduced to $<1 \mathrm{~Pa}$ by means of a differential pumping scheme along three chambers from source to target [22], and the near-surface plasma is considered to be in the strongly-coupled regime [11]. Both the positions of the plasma source and that of the target can be adjusted along a wide range along the centre axis of the machine $(z)$.

Radial profiles of $T_{\mathrm{e}}$ and $n_{\mathrm{e}}$ were measured at a position of $1250 \mathrm{~mm}$ from the source 
(28.5-150 mm from the target) using a Thomson Scattering (TS) system [23] located at $z=0$. The targets used during the plasma emissivity and detachment experiments were $1 \mathrm{~mm}$ thick $\mathrm{W}$ disks of $30 \mathrm{~mm}$ in diameter. The total power transferred to the targets was determined via calorimetry of the cooling water with an accuracy within $10 \%$.

\subsection{Resistive bolometer}

The Magnum-PSI bolometer is a 4-channel resistive metal foil detector from IPT Albrecht. Each sensor of dimensions $1.3 \times 3.8 \mathrm{~mm}^{2}$ is a $5 \mu \mathrm{m}$ thick gold $(\mathrm{Au})$ foil covered with a $150 \mathrm{~nm}$ thick aluminium (Al) heat conducting layer. Two out of four sensors are additionally provided with a $150 \mathrm{~nm}$ thick $\mathrm{C}$ layer to enhance absorption at longer wavelengths. The spectral absorption of this multi-layered sensor is shown in Fig. 1. Further details can be found in Appendix C.

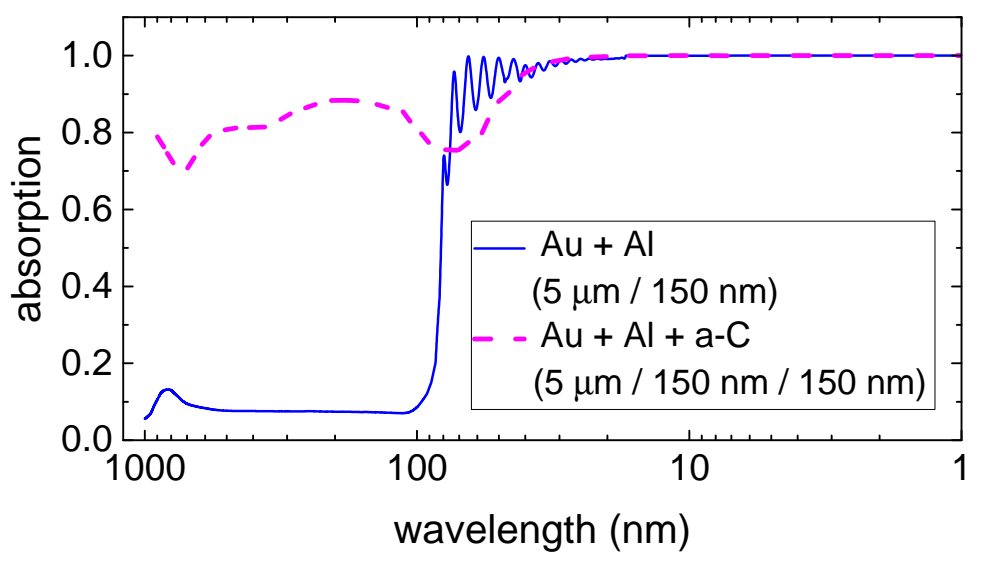

Figure 1: Spectral absorption of both the (uncoated) $\mathrm{Au}+\mathrm{Al}$ and (coated) $\mathrm{Au}+\mathrm{Al}+\mathrm{a}-\mathrm{C}$ bolometer sensors for the range of 1-1000 nm. Details are provided in Appendix C.

The temperature of the sensor rises due to impinging radiation and neutral particles which results in an electrical resistance change in two opposite legs of a Wheatstone bridge circuit, at a theoretical detection limit of $1 \mu \mathrm{W} \mathrm{cm}{ }^{-2}$. The two remaining resistors in the circuit are shielded from radiation in order to correct for temperature drifts of the detector head as a whole. Each sensor is excited by a $U_{\mathrm{pp}}=20 \mathrm{~V}, 20 \mathrm{kHz}$ sine voltage provided by an individual amplifier unit. Any imbalance arising between the heat absorbing and shielded resistors is obtained from the output signal using quadrature detection [15]. The resulting bridge voltage is consequently amplified between 10 and $5000 \mathrm{x}$ and demodulated and filtered by the amplifier unit. The DC output voltage from the amplifier, $U_{\mathrm{b}}$ is send to a digitizer (12-Bit MC USB-200) and finally stored in a PC. The parameter $\Delta U_{\mathrm{b}}$ is defined to be the voltage difference during plasma radiation exposure with respect to the level before (hence a DC imbalance). The power absorbed by each sensor $\left(P_{\mathrm{s}}\right)$ is then obtained from $\Delta U_{\mathrm{b}}$ using the following equation [24], taking into account the sensor sensitivity $S(\mathrm{~V} / \mathrm{W})$ and time constant $\tau(\mathrm{s})$ :

$$
P_{s}=\frac{1}{S}\left(\Delta U_{\mathrm{b}}+\tau \frac{d \Delta U_{\mathrm{b}}}{d t}\right) .
$$


The calibration of $S$ and $\tau$ are done using existing methods [25] and is discussed in Appendix B. The calibration numbers in vacuum conditions (table B1) are consequently applied throughout the current study. Equation 1 is a simplification of the full relation which includes the cable resistance and capacitance [26]. The cable length used in this study is $10 \mathrm{~m}$ which results in a cable resistance equal to $1.1 \%$ of the bolometer input impedance, leading to an error in the radiated power of similar magnitude.

The power incident on the bolometer detector $\left(P_{\mathrm{s}}\right)$ can be related the line-integrated brightness of the plasma $\left(\int \varepsilon d l\right)$ within the bolometer viewing cone using the geometrical constrains set by the aperture size $\left(A_{\mathrm{ap}}\right)$, sensor size $\left(A_{\mathrm{s}}\right)$ and distance from sensor to aperture $\left(l_{\mathrm{s}, \mathrm{ap}}\right)$

$$
P_{\mathrm{s}}=\frac{1}{4 \pi} \frac{\cos ^{2}(\theta) A_{\mathrm{s}} A_{\mathrm{ap}}}{l_{\mathrm{s}, \mathrm{ap}}^{2}} \int \varepsilon d l
$$

where $\theta$ indicates the misalignment of the surface normal of the aperture and sensor surfaces to the line of sight. The latter angle varies maximally $6.1^{\circ}$ which reduces the effective sensor surface by $1.1 \%$ and is neglected during analysis.

\subsection{Field of view}

Projections of the bolometer FOV in the axial and radial direction of the plasma beam in Magnum-PSI are shown in Fig. $2 a$ and $2 b$ respectively.

a)

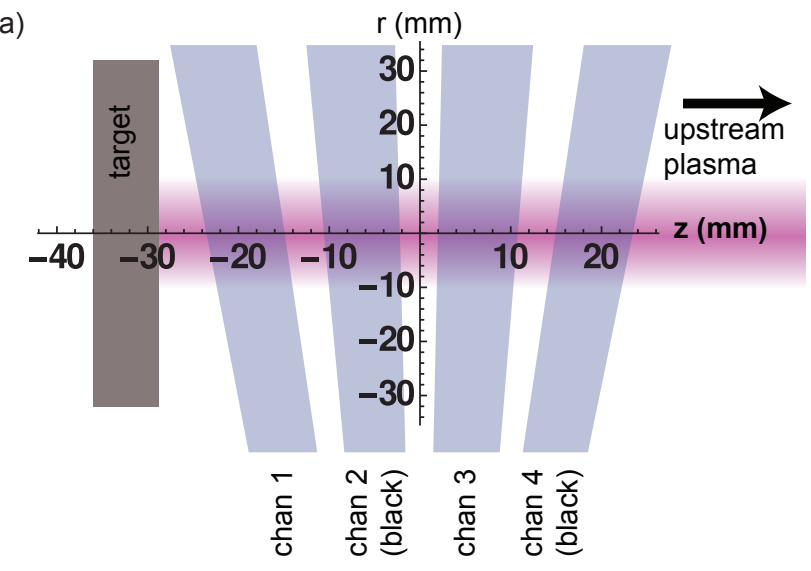

b)

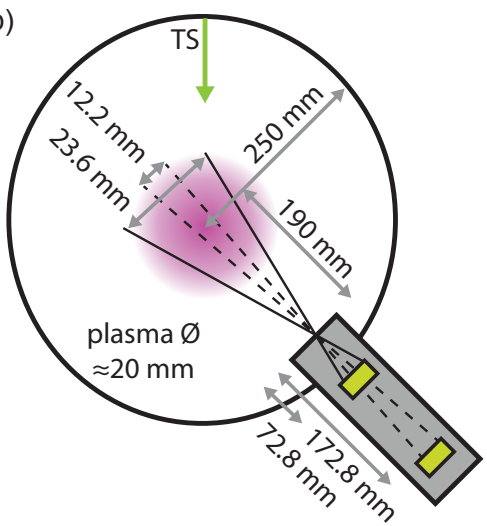

Figure 2: Field-of-view of the bolometer along the axial extent of the plasma beam when $l_{\mathrm{ap}, \mathrm{d}}=72.8 \mathrm{~mm}(a)$. The radial extend of the viewing cone in the case of shortest distance between sensor and aperture is $23.6 \mathrm{~mm}$ (solid lines) and $12.2 \mathrm{~mm}$ at the farthest position (dashed lines) (b). The axial position of the target can be freely adjusted along $z$.

The extent of the observable volume in the radial direction of the beam $(\Delta R)$ as function of the adjustable distance between sensors and aperture, $l_{\mathrm{s} \text {,ap }}=72.8-172.8 \mathrm{~mm}$, can be expressed as

$$
\Delta R=2 h_{d}\left(\frac{l_{\mathrm{p}, \mathrm{d}}}{l_{\mathrm{s}, \mathrm{ap}}}+\frac{1}{2}\right)
$$


with $h_{\mathrm{d}}=3.8 \mathrm{~mm}$ the sensor height and $l_{\mathrm{p}, \mathrm{d}}=190 \mathrm{~mm}$ the distance between the plasma beam centre and the aperture. When the detector is moved closest to the aperture, $\Delta R$ is $23.6 \mathrm{~mm}$ which is similar or slightly larger than the typical FWHM of the plasma beam, depending on operational settings. Its radial viewing width decreases to $12.2 \mathrm{~mm}$ if the bolometer is positioned farthest away from the aperture which allows for better radial resolution at the expense of signal level due to the increased solid angle.

The coordinate $z=0$ corresponds to the TS measurement position [23] where the total observable $z$-range by the bolometer is approximately $50 \mathrm{~mm}$ (Fig. $2 a$ ). It is known that the plasma parameters in Magnum-PSI may change along $z$ when comparing source to target conditions, as is reported [27] from studies in Pilot-PSI, the predecessor of Magnum-PSI. Especially variation in $T_{\mathrm{e}}$ may lead to significant changes in the plasma emission $\varepsilon$. However, given that $z$-range spanned by the bolometer is much smaller than the typical distance from the FOV to the plasma source $(\Delta z=1250 \mathrm{~mm})$ we make the assumption that $T_{\mathrm{e}}$ and $n_{\mathrm{e}}$ are independent of $z$ around the bolometer viewing position which makes the interpretation of the signals by different sensors simpler.

This assumption is no longer tenable when the target surface is brought in close vicinity of the FOV. In such cases, large variations in plasma emissivity along $z$ may be induced due to recycled neutrals and the formation of a pre-sheath. The pre-sheath length in the sheath-limited strongly coupled regime can be approximated by the ionneutral charge exchange mean free path, which is typically $\sim 10 \mathrm{~mm}$ for $\mathrm{H}$ and $\sim 1 \mathrm{~mm}$ for Ar in standard Magnum-PSI conditions with a floating target [28, 29]. Similar radiation levels for each of the 4 sensors are thus to be expected if the target is located at $z \lesssim-35 \mathrm{~mm}$.

\section{Results and discussion}

\subsection{Time-evolution of plasma emission}

The line-integrated emissivity obtained by channels 2 and 3 during a $225 \mathrm{~s}$ plasma exposure of a W sample are shown in Fig. 3. This parameter is evaluated using Eq. 2 and calculating $P_{\mathrm{s}}$ as in Eq. 1 from the increase of the bolometer bridge voltage due to impinging plasma radiation $\left(\Delta U_{\mathrm{b}}\right)$. A linear drift correction has been applied to $\Delta U_{\mathrm{b}}$ by assuming the phase of $\mathrm{Ar}$ emission ( $t=25-110 \mathrm{~s})$ to be constant in time, which is confirmed by current and voltage measurements of the plasma source. Furthermore, an offset correction has been applied by defining $\Delta U_{\mathrm{b}}=0$ at $t=0$ (prior to plasma ignition). The digitizer operated at 100 samples per second and no data smoothing is used.

Several phases during the plasma discharge, distinguished by different settings of the plasma source and position of the target, can be identified when examining the emission evolution in Fig. 3. The magnetic field strength is held constant at $1 \mathrm{~T}$. Firstly, the plasma source is ignited at $t=0$ by feeding $3.2 \mathrm{slm}$ (standard litre per minute, equal to $4.5 \times 10^{20}$ particles s $^{-1}$ ) Ar gas and establishing a source current of $105.5 \mathrm{~A}$. The target is far away from the bolometer FOV at this stage. Secondly, at $t=110 \mathrm{~s}$, the Ar gas flow is 


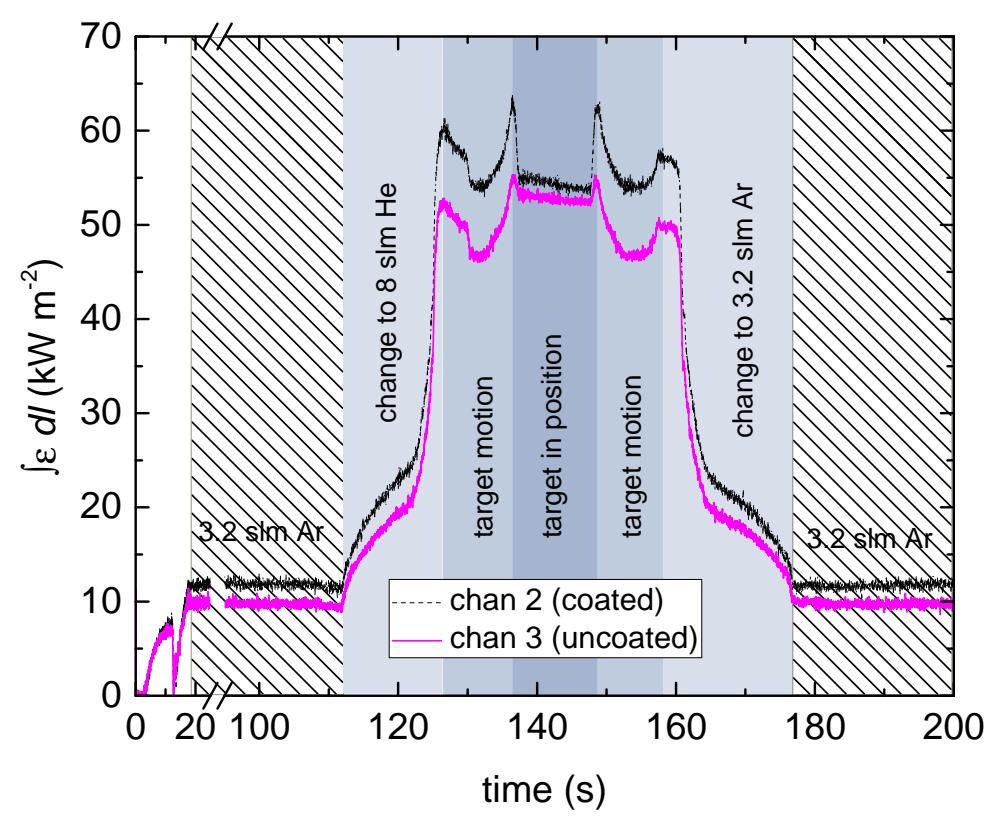

Figure 3: Time evolution of the line-integrated emissivity from the plasma obtained by bolometer channels 2 and 3. The radiated power is strongly affected by the gas species (Ar and He), plasma conditions and presence of the target near the bolometer FOV. The difference in absorbance between channel 2 (coated) and channel 3 (uncoated) can be clearly seen.

reduced while He is gradually injected in the source up to $12 \mathrm{slm}$ and the source voltage adjusted to reach $I_{\mathrm{s}}=126 \mathrm{~A}$. The radiated power clearly increases in this phase. Thirdly, the target is moved just in front of the bolometer FOV $(z=-28.5 \mathrm{~mm})$ at $t=130-149 \mathrm{~s}$. Channel 2 decreases in this phase while channel 3 rises with respect to the signal levels in the preceding phase (target motion). The change in relative signal levels during the near-target measurement phase is explained by a $z$-dependency on the plasma emission near the target: particle recycling and subsequent cooling and recombination of the near-surface plasma reduce the local emission (hence a decrease of channel 2). After retraction of the target at $t \approx 150 \mathrm{~mm}$, the gas flow is gradually switched back to $\mathrm{Ar}$ reaching $3.2 \mathrm{slm}$ at $t=175 \mathrm{~s}$.

From the steady-state emission phase in $\operatorname{Ar}(t=25-110 \mathrm{~s})$ with the target far away from the FOV, the difference in absorbance between channels 2 and 3 is clearly seen. The blackened channel 2 shows a higher absorbance of roughly $22 \%$. Interestingly, the difference between channels 2 and 3 reduces to $\sim 15 \%$ during the phase of pure He emission, likely due to a change of the dominant line emission towards smaller wavelengths than the optical range. The latter increased absorbance factor is similar to values of $13-15 \%$ obtained in the divertor region of TCV [19].

Figure $4 a$ shows another time evolution of plasma emission throughout a $\mathrm{Ar} / \mathrm{H}$ discharge including a period of $\mathrm{N}_{2}$ seeding. The sample rate of the bolometer digitizer was only $10 \mathrm{~Hz}$ and no smoothing has been applied. Nitrogen is injected near the target 


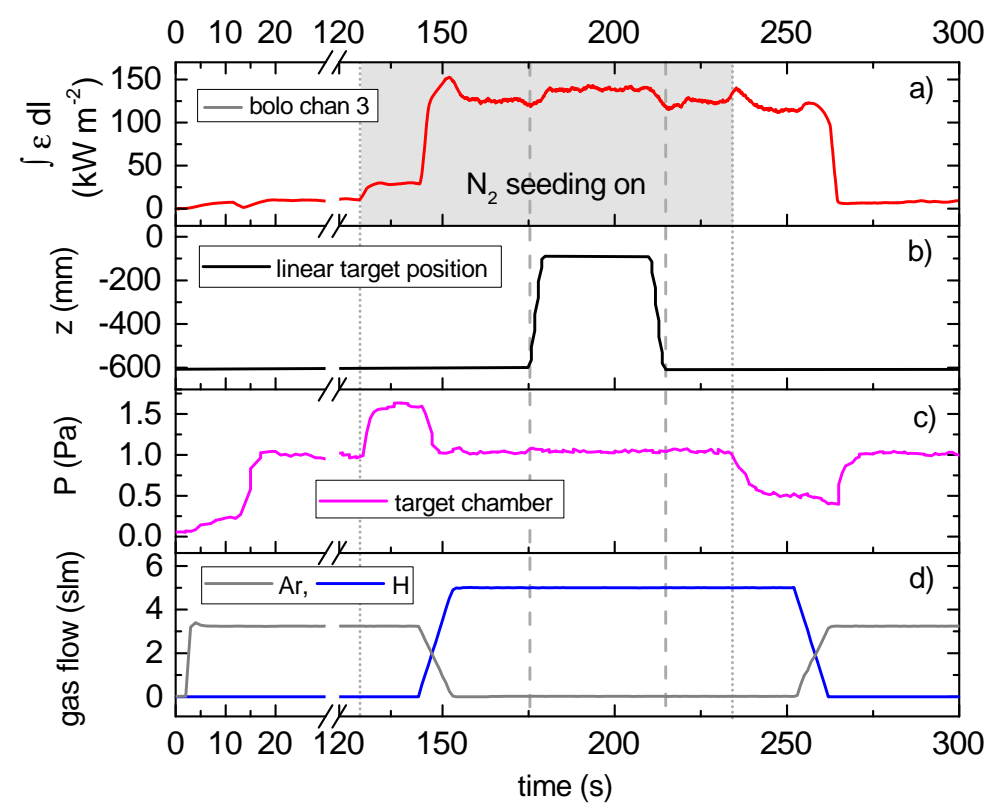

Figure 4: Time-dependent line-integrated emissivity from a $\mathrm{Ar} / \mathrm{H}$ plasma with $\mathrm{N}_{2}$ seeding $(a)$, distance between target and the bolometer FOV $(b)$, pressure in the target chamber $(c)$ and gas flow in the source $(d) . \mathrm{N}_{2}$ is seeded at location $z=0$ for a duration as illustrated by the grey box in panel $a$ and indicated by the dotted lines. The phase where the target is moved close to the bolometer FOV is highlighted by the dashed vertical lines.

chamber wall via a remote controlled pressure valve. The $\mathrm{N}_{2}$ pressure distribution close to the plasma can be considered uniform since the distance from plasma to injection valve is much larger than the plasma beam diameter. The raw bolometer output voltage has again been corrected for linear drifts and an offset voltage has been applied by taking $\Delta U_{\mathrm{b}}=0$ at the phase before igniting the plasmas source (at $t=0-2 \mathrm{~s}$ ). The $z$-position of the target with respect to the bolometer FOV and the pressure in the target chamber are shown in panels $4 b$ and $c$ respectively while panel $d$ shows the gas flow in the source. It is clear that changing from $\mathrm{Ar}$ to $\mathrm{H}$ is accompanied by strongly rising plasma emission which is explained by Molecular Assisted Recombination processes [30, 31] due to the presence of $\mathrm{H}_{2}$ in the plasma beam.

As inferred from $4 c$, the gas pressure in the target chamber temporarily increases as a result of opening the $\mathrm{N}_{2}$ seeding valve, followed by a similar reduction in pressure when the valve is closed. The period of $\mathrm{N}_{2}$ addition is highlighted in Fig. 4 by the grey box and vertical dotted lines. The overall pressure equilibrates at $1 \mathrm{~Pa}$ for the remaining time and is found to be independent of the gas species used. We therefore conclude that the strong increase in bolometer signal is due to the radiated power flux rather than carried by neutrals from the plasma. The effect of the target position on plasma emission is small but measurable, as indicated by the slight increase at $t=175-220 \mathrm{~s}$ (dashed vertical lines). 
The $\mathrm{N}_{2}$ seeding valve is opened at $t=125 \mathrm{~s}$ when an Ar plasma is present, resulting in tripling the radiated power loss. Enhanced radiation by $\mathrm{N}_{2}$ species and/or the potential increase of $n_{\mathrm{e}}$ are expected to account for this, as $T_{\mathrm{e}}$ is likely to drop due to the gas puff which should result in lower emission from both $\mathrm{Ar}$ and $\mathrm{N}_{2}$. The change to $\mathrm{H}$ in the plasma source, initiated at $t \sim 135 \mathrm{~s}$, results in a 5 -fold increase of the line-integrated emission compared to the previous level of $\mathrm{Ar}+\mathrm{N}_{2}$. The presence of $\mathrm{N}_{2}$ in $\mathrm{H}$ enhances the radiated power loss by $\sim 10 \%$ compared to the level without $\mathrm{N}_{2}$ as can be inferred from the decreasing signal upon closing the seeding valve at $t=235 \mathrm{~s}$.

The mechanism driving the increase of radiation during $\mathrm{H}$ exposure in presence of $\mathrm{N}_{2}$ was elucidated by using Eunomia [32], a spatially-resolved 2D Monte-Carlo code developed to simulate the behaviour of neutral particles in the linear plasma devices Pilot-PSI and Magnum-PSI. The code provides the radial atomic $\mathrm{H}$ density as a representation of the recombination yield while accounting for a large number of reaction pathways involving $\mathrm{H}_{2}$ and $\mathrm{N}_{2}$ species [33]. Scenarios with a background pressure of $0.3 \mathrm{~Pa}$ (baseline), $0.5 \mathrm{~Pa}$ (increased $\mathrm{H}_{2}$ pressure) and the combination of $\mathrm{N}_{2}$ and $\mathrm{H}_{2}$ of $0.5 \mathrm{~Pa}$ each are shown in Fig. 5. The peak density and temperature in the centre of the simulated beam was $5 \times 10^{20} \mathrm{~m}^{-3}$ and $5 \mathrm{eV}$ respectively.

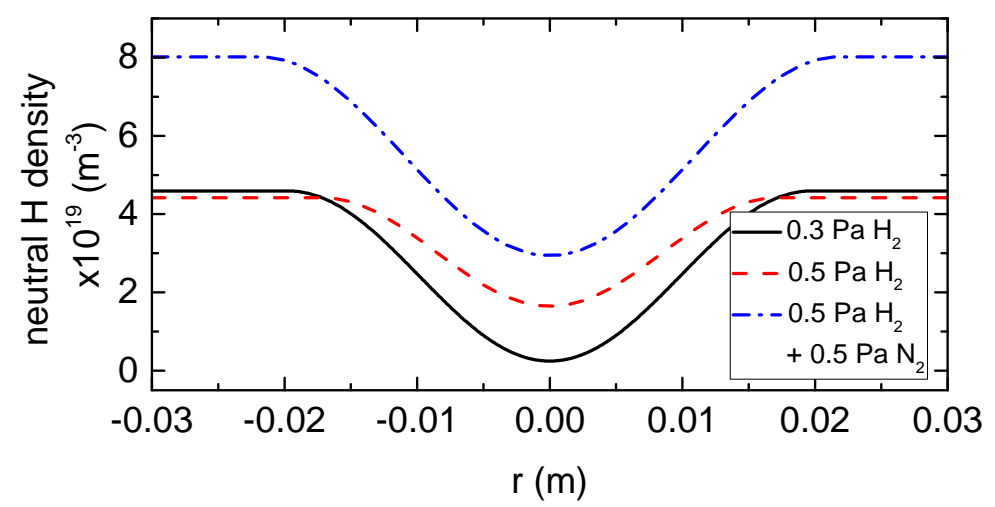

Figure 5: Radial distribution of atomic $\mathrm{H}$ in typical Magnum-PSI plasma conditions at a background pressure of 0.3, 0.5 and $1 \mathrm{~Pa}$ simulated using Eunomia [32]. The addition of $\mathrm{N}_{2}$ clearly leads to an increased level of the atomic $\mathrm{H}$ fraction in the plasma.

Figure 5 shows that increasing the background pressure under the influence of $\mathrm{N}_{2}$ strongly increases the neutral $\mathrm{H}$ density. The formation of atomic $\mathrm{H}$ is facilitated via the presence of $\mathrm{NH}$ molecules which is identified as the main driver of so-called Nitrogen Molecular Assisted Recombination (N-MAR) causing enhanced recombination [33]. As a result, the large atomic $\mathrm{H}$ density increases the fraction of line emission in addition to recombination radiation in the overall radiation yield. We thus conclude that experimentally obtained increase in radiated power loss as a result of $\mathrm{N}_{2}$ seeding in $\mathrm{H}$ can be attributed to MAR processes which are intensified by $\mathrm{H}_{2}-\mathrm{N}_{2}$ chemistry [33]. 


\subsection{Detachment studies}

Figure $6 a$ shows the time evolution of steady-state plasma operation in $\mathrm{H}$ while the background pressure in the target chamber is varied. The target position with respect to the centre of the bolometer FOV as a function of time is shown in panel $6 b$. The background gas pressure in the target chamber is increased up to levels of 4 and $2 \mathrm{~Pa}$ by injecting $\mathrm{H}_{2}$ gas at the wall at a position of $z=0$. The overall background pressure without additional $\mathrm{H}_{2}$ injection is $0.34 \mathrm{~Pa}$. The background pressure level is kept constant during each exposure, as indicated by panel $c$ in Fig. 6. As a result of increasing background pressure, the plasma transitions from sheath-limited conditions to a detached regime characterized by reduced plasma pressure in the near-surface region as measured by TS. The target heat flux reduces as evidenced from calorimetry and a reduction in visible light emission in front of the target.

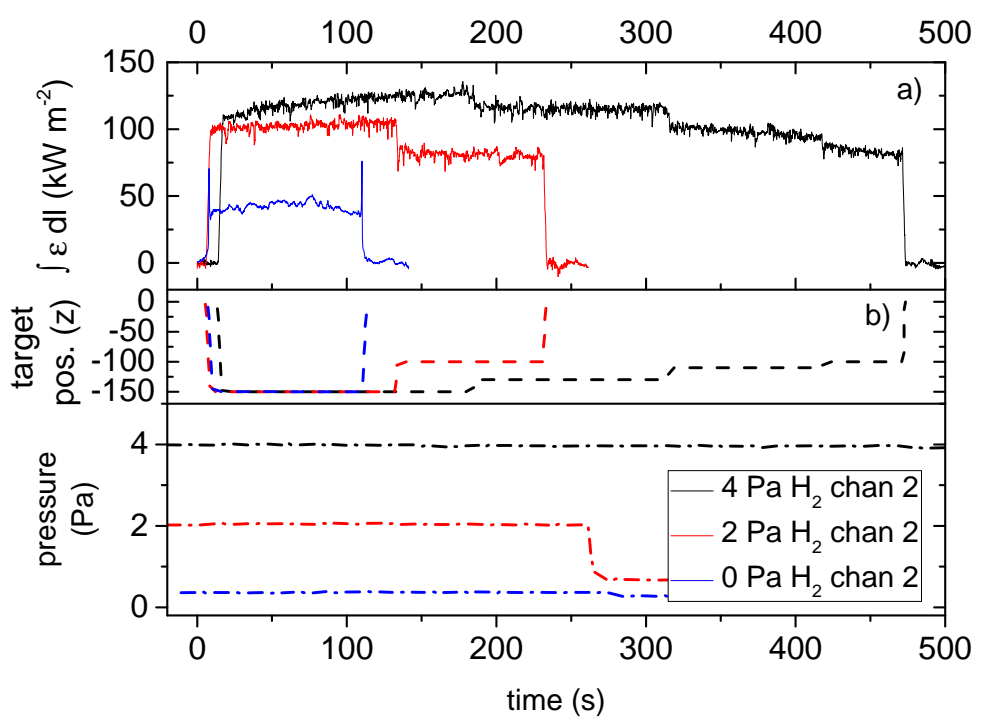

Figure 6: Time evolution of line-integrated emissivity during plasma operation in $\mathrm{H}$ at increased background pressures $(a)$ and position of the target as function of time $(b)$. The background pressure in the target chamber is shown in panel $(c)$. Radiated power loss from the plasma increases with increasing $\mathrm{H}_{2}$ background pressure. The position of the target affects the emissive region and therefore the bolometer measurement. Given that the background pressure remains constant during the bolometer measurement, the drift in the signal (notably at $4 \mathrm{~Pa}$ ) is not attributed to a change in the neutral particle flux hitting the sensor surface.

The target was located at $z=70 \mathrm{~mm}$ each time operational settings were adjusted. This position fully blocks the bolometer detector from receiving radiation while neutrals can still diffuse towards the sensors (Fig. 2). A zero procedure which equilibrates the Wheatstone bridge resulting in $\Delta U_{\mathrm{b}}=0$ is then applied before moving the target to $z=-$ $150 \mathrm{~mm}$ in order to compensate for gas pressure variations which may result from the change in plasma conditions. Caution is normally required when interpreting bolometer 
signals due to possible effects of high-energetic charge exchange (CX) neutrals hitting the sensors [34]. This issue is however expected to be much reduced in Magnum-PSI where the energies of such neutrals are small $\left(T_{\mathrm{n}} \leq T_{\mathrm{i}} \approx T_{\mathrm{e}} \approx 5 \mathrm{eV}\right)$. Active pumping of the Magnum-PSI target chamber [35] which is connected to the bolometer housing by additional air vents next to the aperture (see Appendix A), further mitigates parasitic heating of the sensors due to neutral impact.

When molecules are involved (e.g. $\mathrm{H}_{2}$ ) the formation of neutrals is generally aggravated by a process of ion conversion $\left(\mathrm{H}^{+}+\mathrm{H}_{2} \rightarrow \mathrm{H}_{2}{ }^{+}+\mathrm{H}\right)$ followed by dissociative recombination $\left(\mathrm{H}_{2}{ }^{+}+\mathrm{e} \rightarrow \mathrm{H}^{+}+\mathrm{H}+\mathrm{e}\right)$. Contrary to previous considerations, if such neutrals are ballistic, they may contribute to parasitic heating of the sensors as one cannot directly discern them from radiation. Also, the formation of molecules facilitated by the surface may contribute to the release of energy. Future modelling studies are to be performed where the fraction of ballistic neutrals (possibly heating the sensor) versus thermalized neutrals (accounted for by an offset correction) are determined. In this way, the fraction of radiation can be discriminated from neutral heating.

The electron densities in the cases of 4, 2 and $0.34 \mathrm{~Pa}$, are $1.5,1.1$ and $1.0 \times 10^{20} \mathrm{~m}^{-3}$ respectively while $T_{\mathrm{e}}$ is $0.5,1$ and $2.1 \mathrm{eV}$. Increasing the background pressure to $2 \mathrm{~Pa}$ by $\mathrm{H}_{2}$ gas puffing causes the emission to increase by a factor 2.2 as compared to the reference case without adding gas. This is attributed to both increased line emission from excited neutrals and enhanced recombination radiation since $T_{\mathrm{e}}$ decreases from 2.1 to $1 \mathrm{eV}$ which means that the plasma changes from an ionization dominated to a recombination dominated regime at the mentioned typical density. Doubling the background pressure again to $4 \mathrm{~Pa}$ only increases the radiated power by a factor 1.2 with respect to the level at $2 \mathrm{~Pa}$ background pressure. The modest increase in radiation resulting from the latter step is explained by increased neutral line emission as the plasma already resides in a recombination dominated state as opposed to the change from $0.34 \mathrm{~Pa}$ to $2 \mathrm{~Pa}$.

The stepwise reduction in emissivity over the course of time for the cases of 4 and $2 \mathrm{~Pa}$ are due to the changing target position. In detached conditions, $T_{\mathrm{e}}$ near the target is strongly reduced as opposed to attached conditions. In line with visible camera observations, the region of maximum brightness moves away from the target surface while this is highest just in front of the target surface during sheath-limited conditions. For this reason, moving the target closer to the bolometer FOV results in reduced line-integrated emissivity as observed in Fig. 6.

Although the bolometer functioning is designed to be immune to thermal excursions of the detector head, slowly drifting signals at presumably constant plasma irradiation were observed as can be clearly seen in the H plasma exposure at a duration of $t>450 \mathrm{~s}$ in Fig. 6. The bolometer detector in its current design is thermally isolated and did not have a shutter installed. The temperature of the detector housing thus slowly increases due to a non-uniform areal power flux centered on the active sensor array by radiation through the aperture. Due to differences in path length between the passive and active sensor arrays with respect to the region of power input (centered on the active array), 
it is hypothesized that a slight imbalance between the active and passive legs in the Wheatstone bridge arises which causes a drift and/or offset in the signal. The heat source in this case is radiation only since the background pressure is observed to remain constant over the course of the exposure and could hence not have caused the timedependent drift.

This issue is not present during most bolometer applications on current tokamaks where the pulse duration is in the order of seconds or less and front-end heating of the detector housing by radiation is negligible. Resistive bolometry operation in future fusion devices operating at long pulse lengths may however encounter this effect. Also, the calibration constants may slightly change as a result of thermal drifts which is not taken into account so far. Improving the thermal stability of the detector and rebalancing each channel on a regular time base by blocking the impinging light via a shutter can help mitigate the drift issue. Periodic remote calibration may improve accuracy during long time-scale exposures. The aforementioned issues can be solved by utilizing novel FPGA-based bolometer electronics [36] in combination with the installation of a shutter system, both foreseen to be implemented in the near future.

\subsection{Volumetric plasma emission and comparison to synthetic diagnostic}

Radiated power loss from $\mathrm{H}, \mathrm{He}$, Ne and Ar plasmas in Magnum-PSI was experimentally investigated for $T_{\mathrm{e}}$ and $n_{\mathrm{e}}$ ranging $1-6 \mathrm{eV}$ and $0.3-25 \times 10^{20} \mathrm{~m}^{-3}$ respectively in the centre of the plasma beam. Measurements were carried out during steady-state plasma conditions while the target was positioned at $z=-70 \mathrm{~mm}$, allowing for an almost constant axial emission profile around the bolometer FOV. Prior to the measurement, the target fully obstructed the bolometer FOV while neutrals from the plasma could diffuse towards the sensor in order to set $U_{\mathrm{b}}=0$, thus cancelling the influence of (non-ballistic) neutrals on the bolometer signal.

A 0D time-independent collisional radiative model (CRM) provided by ADAS [18] was applied in parallel to calculate the radially resolved plasma emissivity $\varepsilon(r)$ (in $\mathrm{W} \mathrm{m}^{-3}$ ) in the Magnum-PSI plasma beam. Radial profiles of $n_{\mathrm{e}}$ and $T_{\mathrm{e}}$ measured at $z=0$ by TS simultaneous to the bolometer measurement were fitted by Gaussian functions serving as inputs to this model. ADAS photon emissivity coefficients (PEC values in $\mathrm{W} \mathrm{cm}^{3}$ ) were calculated for a range of suitable $n_{\mathrm{e}}$ and $T_{\mathrm{e}}$ values using the ADAS405 routine which simultaneously solves the time-independent atomic state population distribution at specified conditions taking ADAS ADF11 data as input [18]. The PEC values were subsequently interpolated by both the radial values of $T_{\mathrm{e}}$ and $n_{\mathrm{e}}$ in the plasma beam. The obtained radial PEC value is then multiplied by $n_{\mathrm{e}}(r)$ and the density of radiating atoms $n_{\mathrm{a}}(r)$ (sum of neutrals and ions) obtained from the (local) atomic population distribution provided by the CRM, yielding $\varepsilon(r)=P E C(r) n_{\mathrm{e}}(r) n_{\mathrm{a}}(r)$.

A number of synthetic sight lines from bolometer detector to the plasma is then considered, of which the angle with respect to the centre line is varied between 0 and $2.95^{\circ}$, set by ray tracing for the case of $l_{\mathrm{s}, \text { ap }}=72.8 \mathrm{~mm}$. The power density collected by 
each unique synthetic viewing cord $Q_{\mathrm{i}}$ is consequently found by summing the product of the path length $d l$ (distance between radial coordinates) and the local emissivity $\varepsilon(r)$ over the entire length of the viewing cord traversing the plasma: $Q_{\mathrm{i}}=\sum_{\mathrm{i}} \varepsilon(r) d l$.

In this way, the integral $\int \epsilon d l$ which appears in Eq. 2 can be approximated for each synthetic line of sight. Power incident on the detector for each viewing cord easily follows by inserting the hardware dimensions in this equation while unity spectral absorption of the radiation by the sensor is assumed for simplicity. Averaging over the total number of viewing chords finally yields the total power incident on the detector $\left(P_{\mathrm{s}}\right)$. This procedure is repeated for all gas species and plasma conditions where a simultaneous measurement of bolometry and TS was performed.

The comparison of power incident on the bolometer detector (in $\mathrm{mW}$ ) is compared to the results from the synthetic diagnostic based on the same plasma parameters in Fig. 7. The dashed line indicates a 1:1 agreement. Considering the practical noise equivalent power density of $0.1 \mathrm{~W} \mathrm{~m}^{-2}$, a SNR of 10 (He) to $37(\mathrm{Ar})$ is obtained. The match

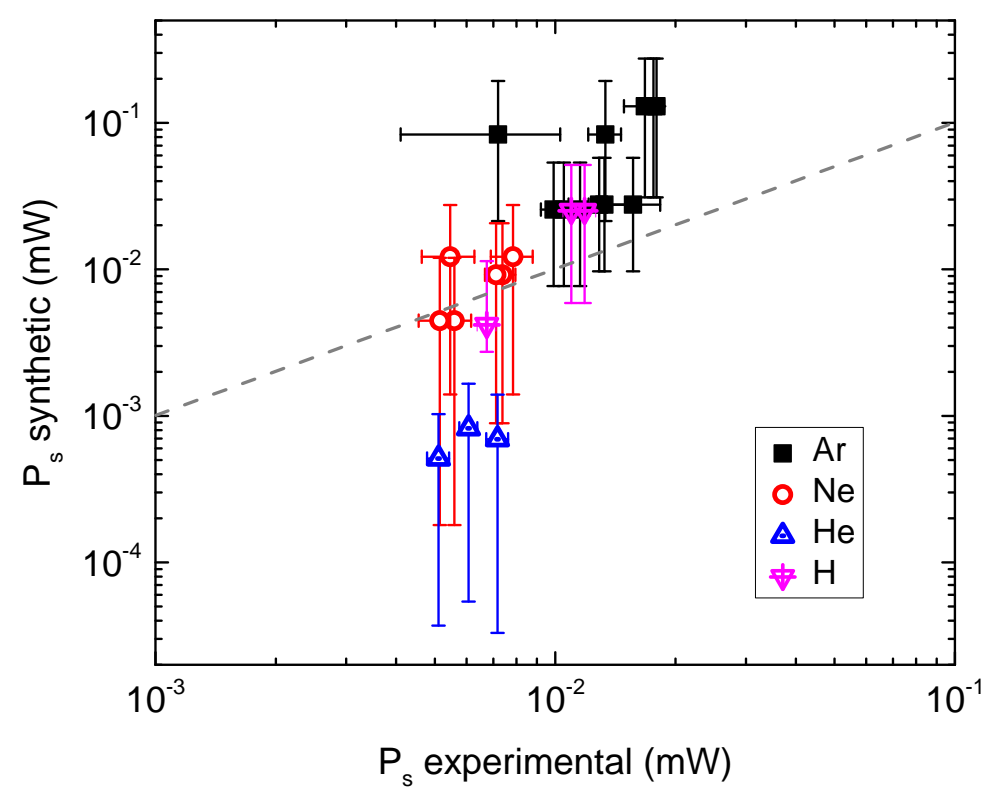

Figure 7: A comparison between the average power received by the bolometer detector and expected incident power from a synthetic diagnostic. Expected radiation levels are calculated with a CRM from the ADAS package while taking $T_{\mathrm{e}}$ and $n_{\mathrm{e}}$ measured by TS as inputs.

between the synthetic diagnostic and the measurements are found to be in agreement within error for $\mathrm{H}$ and Ne species while the bolometer measurement for He is overall 10 times higher than predicted by modelling. In contrast, the measurement of the power incident on the sensor during Ar plasma exposure is a factor 5 lower than predicted. A wide range of emission levels at our conditions is expected based on atomic physics considerations where differences by an order of magnitude in the cooling functions of $\mathrm{He}, \mathrm{Ne}$ and Ar species at similar values of $T_{\mathrm{e}}$ are present [37, 38]. This behaviour is however not reflected in the measured values of power incident on the detector. 
In order to explain this apparent discrepancy, it should be realized that non-coronal radiation, plasma opacity effects, charge exchange recombination and plasma transport phenomena affecting radiation are not taken into account in the model described above. Given the strong dependency of the emissivity on the plasma parameters, a small error in the TS measurements, possibly due to misalignment, results in significant changes of the predicted detector power. Lastly, the role of wavelength-dependant sensor reflectivity and ballistic neutrals are also not taken into account. Hence, given the rudimentary nature of the model, the deviation between measured and predicted values for the case of $\mathrm{He}$ and $\mathrm{Ar}$ are believed to be within limits to conclude that resistive bolometry in Magnum-PSI is found effective in assessing total radiated power loss from the plasma.

\subsection{Power balance}

The previously determined values of $P_{\mathrm{s}}$ and thus $\int \varepsilon d l$ via Eq. 2 yields the possibility of assessing the total power balance in Magnum-PSI. Assuming homogeneous emission over the length $\left(z_{\text {tot }}\right)$ of the plasma beam, the total power loss via radiation $\left(P_{\text {rad }}\right)$ can be calculated as

$$
P_{\text {rad }}=\frac{1}{4} \pi d_{\mathrm{p}} z_{\text {tot }} \int \varepsilon d l,
$$

where $d_{\mathrm{p}}$ represents the diameter of the plasma beam. The plasma beam length was $1250 \mathrm{~mm}$ while $d_{\mathrm{p}}=\mathrm{FWHM}$, obtained from the radial $n_{\mathrm{e}}$ distribution.

The total radiated power obtained in this way is a conservative number, given that the plasma parameters, particularly $T_{\mathrm{e}}$, are known to decrease downstream relative to the upstream conditions. The width of the beam increases at the same time due to radial diffusion, yielding $P_{\text {rad }}$ to increase typically by a factor 3.5 when taking the variation of $T_{\mathrm{e}}$ and $n_{\mathrm{e}}$ along the beam path into account.

The plasma input power achieved by the Magnum-PSI plasma source is approximated by $P_{\text {in,source }}=I \times V-P_{\text {calo,source }}$ where $I$ and $V$ represent the source current and voltage respectively while $P_{\text {calo,source }}$ is the power transferred to the cooling water in the source. Quantifying the efficiency of power transferred to the plasma as function of $P_{\text {in,source }}$ is however complex, partly due to the fact tot heating occurs both inside and outside of the source due to Ohmic dissipation by electric currents carried by the plasma [39]. The source energy efficiency is only previously determined for the case of $\mathrm{H}$, yielding $\sim 4 \%[40]$.

Give the unknown source efficiency for gasses other than $\mathrm{H}$, we now consider a simplified picture of the power balance. When neglecting power loss through particle diffusion out of the magnetized beam, the power balance can be expressed as $P_{\mathrm{in}, \text { source }}=$ $P_{\text {radiation }}+P_{\text {calorimetry }}$ where $P_{\text {in,source }}$ and $P_{\text {radiation }}$ represent the input power and radiative losses respectively and $P_{\text {calorimetry }}$ the power transferred to the target.

Figure $8 a$ shows their apparent dependencies which yields a source efficiency of $35 \%, 39 \%$ and $26 \%$ for the cases of $\mathrm{Ar}, \mathrm{Ne}$ and He respectively. These numbers are expectedly much larger than in the case of $\mathrm{H}$ because no dissociation of molecules is 

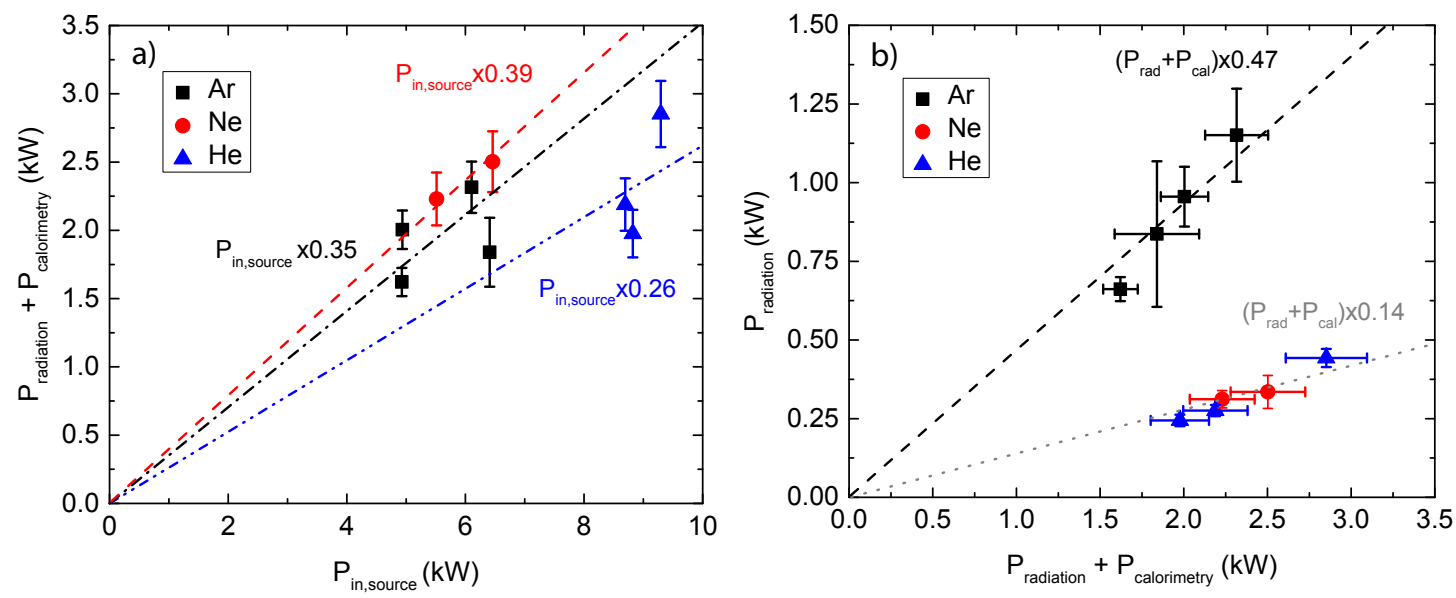

Figure 8: Lost power (radiation and calorimetry) versus source input power (a). Fraction of radiated power loss relative to the sum of radiation and calorimetry (b).

involved. Figure $8 b$ shows $P_{\text {radiation }}$ as function of $P_{\text {radiation }}+P_{\text {calorimetry }}$ for the gas species Ar, Ne, He. The radiated power loss increases with rising total beam power and is a fixed fraction of the input power: $\sim 47 \%$ for $\mathrm{Ar}$ and $\sim 14 \%$ for He and Ne. Also, the radiative loss at similar input power is larger for Ar than Ne which is in agreement with increased expected emissivity of the former based on theory.

The applicability of the bolometer for power balance purposes is demonstrated. However, the absolute magnitude of the radiated power fraction obtained in this fashion is likely to be an overestimation of the real fraction given that particle diffusion exiting the plasma beam (a power loss mechanism) is not taken into account yet. Future studies should compare bolometric measurements of the total radiated power to the difference between total power in the beam between source and target position. This requires TS measurements at target and source position at equal plasma conditions with bolometric analysis which is in principle possible in Magnum-PSI.

\section{Conclusion and outlook}

A 4-channel resistive bolometer, capable of measuring total radiated power loss from the plasma, has been successfully deployed on Magnum-PSI, which marks the first demonstration of this sort of diagnostic on a linear plasma device. The detector is equipped with two carbon-coated sensors out of a total of four viewing the plasma perpendicularly, hereby providing an axially resolved measurement around the Thomson Scattering position. The observed region can be varied from a diameter of 12.2 to $23.6 \mathrm{~mm}$ by linearly adjusting the detector position, giving the capability to observe the entire beam diameter. Time-traces of steady-state plasma radiation over the course of $>450 \mathrm{~s}$ are obtained, hereby encountering voltage drift effects attributed to thermal excursions of the detector which may be expected similarly during application of resistive 
bolometers in future reactors operating at long timescales. Measurements of the power lost via radiation from $\mathrm{H}, \mathrm{He}$, Ne and Ar plasmas using standard settings in MagnumPSI yielded sensor power densities of 10-37 times the practical noise-equivalent power density of $\sim 0.1 \mathrm{~W} \mathrm{~m}^{-2}$. Increasing the background pressure by $\mathrm{H}_{2}$ and $\mathrm{N}_{2}$ seeding greatly increased the radiated power density to maximally $\sim 125 \mathrm{~kW} \mathrm{~m}^{-2}$. A comparison of measured radiated power levels to those obtained from a synthetic diagnostics based on ADAS CRM calculations, yielded good agreement for $\mathrm{H}$ and $\mathrm{Ne}$ species whereas the prediction for Ar emission was too high $(\times 5)$ and for $\mathrm{He}$ too low $(\times 10)$. The obtained emissivity values were used to make a rough estimation of the power balance in Magnum-PSI, resulting in maximally $45 \%$ and $14 \%$ lost power through radiation by $\mathrm{Ar}$ and $\mathrm{Ne} / \mathrm{He}$ respectively assuming only power loss through radiation and energy transfer to the target.

The Magnum-PSI bolometry system is demonstrated to be a valuable diagnostic in studies of power balance and divertor detachment studies. Given that the system is capable of providing axial resolved scans of the radiated power profile in front of a target, new plasma-surface interaction science can be performed, for instance in assessing the total radiated power fraction during vapour shielding of divertor targets.

A current upgrade of the system involves the installation of a shutter located just in front of the detector, blocking radiation but allowing for gas transfer which eases the bridge rebalancing procedure and increases measurement accuracy. In addition, a frequently performed remote calibration of the sensitivity and time constant in changing plasma conditions, hereby correcting for parasitic effects due to neutrals, can be performed using new FPGA-based electronics.

\section{Acknowledgements}

DIFFER is part of the Netherlands Organisation for Scientific Research (NWO). The work has been carried out within the framework of the EUROfusion Consortium and has received funding from the Euratom research and training programme 2014-2018 under grant agreement No 633053. The views and opinions expressed herein do not necessarily reflect those of the European Commission. DIFFER is a partner in the Trilateral Euregio Cluster TEC.

\section{Appendix A. Hardware design of bolometer housing on Magnum-PSI}

An exploded CAD drawing of the bolometer housing designed for Magnum-PSI is shown

in Fig. A1. The entire structure is mounted on the S3-225 port which is positioned at an equal axial distance from the plasma source as the TS system such that the measurement region of the bolometer incorporates the TS measurement position. Inside the tube, the bolometer sensor array is attached to four arms only fixed to the rear flange which also contains the electrical feedthroughs for the sensor cables (drawn separately in the bottom of Fig. A1). The rear flange on its turn is attached to a $100 \mathrm{~mm}$ 


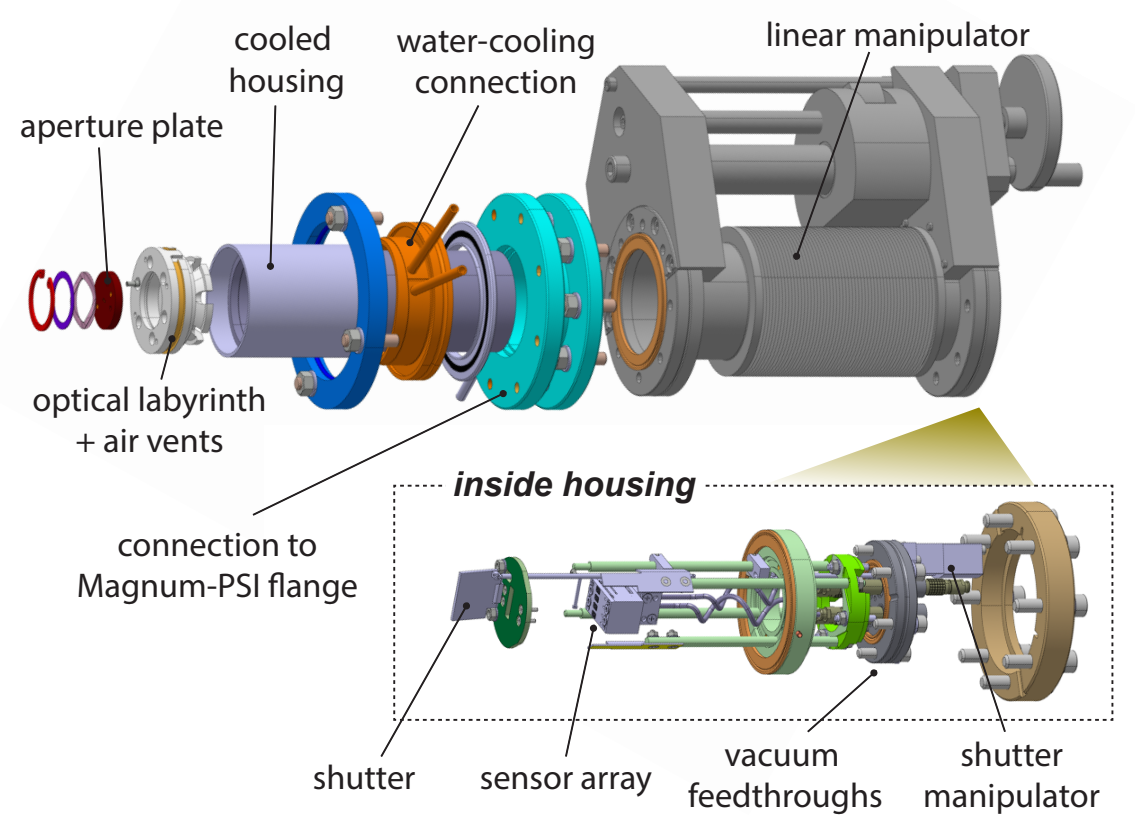

Figure A1: The 4-channel resistive bolometer detector is mounted on a linear manipulator arm in a water-cooled tube attached the Magnum-PSI target chamber. The distance between aperture and the plasma beam centre is $190 \mathrm{~mm}$ while the distance between aperture and sensor can be varied between 72.8 and $127.8 \mathrm{~mm}$. Internal reflections in the bolometer housing are minimized by blackened and textured surfaces surrounding the detector.

linear translation manipulator, hereby providing the possibility of adjusting the distance between sensor and aperture $\left(l_{\mathrm{s}, \text { ap }}\right)$ from $72.8 \mathrm{~mm}$ to $172.8 \mathrm{~mm}$. Bringing the aperture ( $A_{\mathrm{ap}}=1.3 \times 3.8 \mathrm{~mm}^{2}$, equal to size of a sensor) closer to the plasma region increases the solid angle and thus the signal level, at the expense of spatial resolution (see Fig. 2b). For the same reason, the aperture plate is mounted on a tube which intrudes into the Magnum-PSI target chamber by $60 \mathrm{~mm}$, yielding a fixed distance of $190 \mathrm{~mm}$ between the centre of the plasma column and the aperture. The water-cooled tube between aperture and detector is entirely blackened (using hot chemical blackening) and textured in order to minimize internal reflections. As well as the aperture opening, additional air vents provided with an optical labyrinth structure allows the pressure inside the bolometer housing to be readily equilibrated with the pressure in the target chamber. The shutter and shutter manipulator drawn in Fig. A1 are part of a planned upgrade.

\section{Appendix B. Calibration of the bolometer sensor}

The sensitivity $S$ is obtained from presenting an Ohmic heating pulse of known power to the bolometer while measuring the voltage increase $\Delta U_{\mathrm{b}}$ [25]. The time constant is obtained from fitting an exponential decay function to $\Delta U_{\mathrm{b}}(t)$ after transiently heating the bolometer sensor with a pulsed laser $(0.7 \mathrm{~mW}, 532 \mathrm{~nm})$. The calibration constants 
for each sensor at both atmospheric pressure and at $10^{-3} \mathrm{~Pa}$ are shown in table B1. The

\begin{tabular}{c|c|clll}
\hline \multirow{2}{*}{ chan } & blackened & air & \multicolumn{3}{c}{ vacuum } \\
\hline 1 & no $(\mathrm{V} / \mathrm{W})$ & $\tau(\mathrm{ms})$ & $\mathrm{S}(\mathrm{V} / \mathrm{W})$ & $\tau(\mathrm{ms})$ \\
\hline 2 & yes & $10.0 \pm 0.16$ & $80.5 \pm 1$ & $16.1 \pm 0.1$ & $148.5 \pm 1.2$ \\
3 & no & $10.7 \pm 0.2$ & $86.8 \pm 2.4$ & $16.1 \pm 0.1$ & $150.1 \pm 2.4$ \\
4 & yes & $10.8 \pm 0.2$ & $86.8 \pm 1.8$ & $15.9 \pm 0.1$ & $149.8 \pm 5.9$ \\
& & & & &
\end{tabular}

Table B1: Bench-top calibration values of the different bolometer channels at atmospheric pressure and $10^{-3} \mathrm{~Pa}$.

time resolution of the diagnostic is $\sim 100 \mathrm{~Hz}$ as determined in a similar setup [17] but ultimately depends on the incident power density. Transient response is not addressed and only results obtained in steady-state plasma conditions relative to the bolometer time resolution are discussed here.

\section{Appendix C. Sensor absorbance}

The standard IPT bolometer sensors are $5 \mu \mathrm{m}$ thick Au foils provided with a $150 \mathrm{~nm}$ Al heat conducting layer. This type of sensor exhibits approximately unity spectral absorbance for wavelengths $<100 \mathrm{~nm}$ [41] where most of the plasma radiated power is emitted, especially in a tokamak environment. A significant fraction of plasma emission in Magnum-PSI is however expected to occur in the visible wavelength range, given that $T_{\mathrm{e}} \approx 1-5 \mathrm{eV}[10]$. The increase in reflectivity of the $\mathrm{Au}+\mathrm{Al}$ sensor at longer wavelengths can lead to an underestimation of the radiated power in particularly divertor-like plasma conditions [19]. Hence, In order to enhance absorption by the foils at the lower end of the photon energy spectrum, bolometer sensors 2 and 4 have been coated by a thin layer (maximally $150 \mathrm{~nm}$ ) of carbon (99.99\% purity) via DC magnetron sputter deposition at room temperature.

This thickness must be regarded as an upper limit valid in the centre of the foil since the thickness around the edges is expected to be lower due to partial shielding by the mask during deposition. The bolometer detector and blackened sensors are shown in Fig. C1. No difference in the calibration constants $S$ and $\tau$ due to addition of the coating has been found as can be inferred from table B1.

The theoretical spectral absorption of both the $\mathrm{Au}+\mathrm{Al}$ sensors (channel 1 and 3) and $\mathrm{Au}+\mathrm{Al}$ with amorphous carbon (channels 2 and 4) are shown in Fig. 1 in section 2.2 for the range of $0.1-1000 \mathrm{~nm}$. Data is obtained from a simulation of specular reflection $R_{\mathrm{s}}$ for two simulated layered samples, with perfect interfaces, i.e. without layer intermixing or surface roughness [42]. The absorption is approximated by $1-R_{\mathrm{s}}$ since transmission is regarded negligible for the wavelength range of $1-1000 \mathrm{~nm}$ as shown in Fig. 1. Vacuum is assumed above the first layer and below the last. The angle of incidence of the incident 


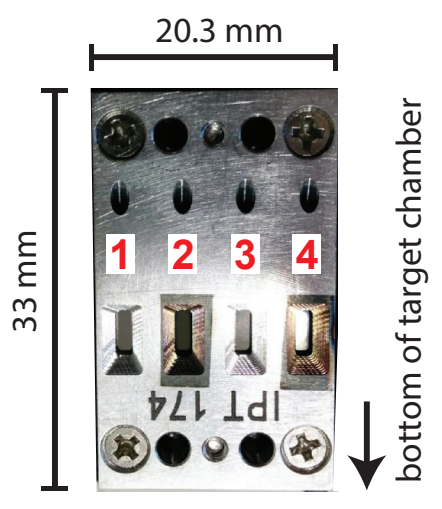

Figure C1: The bolometer detector head with channel numbers as indicated. A $150 \mathrm{~nm}$ thick layer of carbon is applied to channels 2 and 4 . The bottom of the detector is oriented towards the bottom of the Magnum-PSI target chamber.

unpolarized beam was 1.5 degrees with respect to the surface normal, which is similar to the bolometer lines of sight. Optical constants for larger wavelengths were partly used from experimental measurements [43] while these at shorter wavelengths were derived from experimental atomic scatter factors [44] with densities of $2.2,3.26,19.32 \mathrm{~g} \mathrm{~cm}^{-3}$ for the $\mathrm{a}-\mathrm{C}, \mathrm{Al}$ and $\mathrm{Au}$ layers respectively.

The increase in absorption for the wavelength range 100-1000 $\mathrm{nm}$ due to addition of a $\mathrm{C}$ coating is evident from Fig. 1. Although the radiated power loss from the plasma in divertor conditions is predominantly carried by photons with shorter wavelengths [19], the addition of the $\mathrm{C}$ coating is deemed necessary for accurately determining total radiated power loss in such an environment.

\section{References}

[1] Maisonnier D, Campbell D, Cook I et al. 2017 Nucl. Fus. 47 1524-1532

[2] Zohm H 2013 Fus. Eng. Des. 88 428-433

[3] Pitts R, Carpentier S, Escourbiac F et al. 2013 J. of Nucl. Mat. 438 S48 - S56

[4] Krasheninnikov S, Kukshkin A, Lee W et al. 2017 Nucl. Fus. 57102010

[5] Kallenbach A, Bernert M and Dux R 2013 Plas. Phys. Contr. Fus. 55124041

[6] TW Morgan P Rindt G v E et al. 2018 Plas. Phys. Contr. Fus. 60014025

[7] Abdou M, Ying A, Morley et al. 2001 Fus. Eng. Des. 54 181-247

[8] van Eden G, Morgan T W, Aussems D U B, van den Berg M A, Bystrov K and van de Sanden M C M 2016 Phys. Rev. Lett. 116(13) 135002

[9] van Eden G, Kvon V, van de Sanden M et al. 2017 Nat. Comms. 8(192)

[10] van Eck H, Kleyn A and Lof A 2012 Appl. Phys. Lett. 101224107

[11] Temmerman G D, van den Berg M, Scholten J et al. 2013 Fus. Eng. Des. 88 483-487

[12] Kukushkin A, Pacher H, Loarte A et al. 2009 Nucl. Fusion 49075008

[13] Ohno N 2017 Plas. Phys. Contr. Fus. 59034007

[14] Hayashi Y, Jesko K, van der Meiden H et al. 2016 Nucl. Fus. 56126006

[15] Mast K, Vallet J, Andelfinger C et al. 1991 Rev. Sci. Instrum. 62744

[16] Bernert M, Eich T, Burckhart A et al. 2014 Rev. Sci. Instrum. 85033503 
[17] van Eden G, Reinke M, Peterson B et al. 2016 Rev. Sci. Instrum. 87 11D402

[18] Summers H 2004 The ADAS User Manual - http://www.adas.ac.uk version 2.6

[19] Sheikh U, Duval B, Labit B et al. 2016 Rev. Sci. Instrum. 87

[20] Vijvers W, Schram D and Shumack A 2010 Plasma. Sources. Sci. Tech. 19065016

[21] van Eck H et al. 2017 Proc. MT-25 Conf. 2017 submitted

[22] Scholten J, Zeijlmans van Emmichoven P, van Eck H et al. 2013 Fus. Eng. Des. 88 1785-1788

[23] van der Meiden H, Al R, Barth C et al. 2008 Rev. Sci. Instrum. 79013505

[24] Murari A, Mast K, D'ambra L et al. 1995 Rev. Sci. Instrum. 66665

[25] Murari A, Cecconello M, Marrelli L et al. 2004 Rev. Sci. Instrum. 75 2692-2699

[26] Giannone L, Mast K and Schubert M 2002 Rev. Sci. Instrum. 733205

[27] Vijvers W, Al R, Cardozo N L et al. 2007 Proc. 28th Int. Conference on Phenomena in ionized Gases 11 1793-1796

[28] Janev R, Reiter D and Samm U 2003 Collision processes in low-temperature hydrogen plasmas (academia.edu)

[29] Rapp D and Francis W 1962 J. of Chem. Phys. 54 2631-2645

[30] Ohno N, Ezumi N, Takamura S et al. 1998 Phys. Rev. Lett. 81 818-821

[31] Pigarov A 2002 Phys. Scr. T96 16-31

[32] Wieggers R 2012 PhD thesis Eindhoven University of Technology

[33] Perillo R, Chandra R, Akkermans G et al. 2018 submitted to Plasma. Phys. Contr. Fus.

[34] Verbeek H, Stober J and Coster D 1998 Nucl. Fusion 381789

[35] van Eck H, Hansen T, Kleyn A et al. 2011 Plasma Sourc. Sci. Tech. 20045016

[36] Lovell J, Naylor G, Field A and otheres 2016 Rev. Sci. Instrum. 87 11E721

[37] Mavrin A 2017 J. Fus. Energ. 36 161-172

[38] Reinke M, Meigs A, Delabie E et al. 2017 Nucl. Mater. and Energy 12 91-99

[39] de Groot B et al. 2007 Fusion Eng. Des. 82 1861-1865 ISSN 09203796

[40] Vijvers W, van Gils C, Goedheer W et al. 2008 Physics of Plasmas 15093507

[41] Huber A, McCormick K, Andrew P et al. 2007 Fus. Eng. Des. 82 1327-1334

[42] Huber S, van de Kruijs R, Yakshin A et al. 2014 Optics Express 22490

[43] Palik E 1985 Handbook of Optical Constants of Solids (Academic Press, Inc.)

[44] http://henke.lbl.gov/optical_constants/asf.html 